\title{
SUBMILLIMETER POLARIZATION SPECTRUM IN THE VELA C MOLECULAR CLOUD
}

\author{
Natalie N. Gandilo ${ }^{1,2}$, Peter A. R. Ade ${ }^{3}$, Francesco E. Angilè ${ }^{4}$, Peter Ashton ${ }^{5}$, Steven J. Benton ${ }^{6,7}$, Mark J. Devlin ${ }^{4}$, \\ Bradley Dober ${ }^{4}$, Laura M. Fissel ${ }^{5}$, Yasuo Fukui ${ }^{8}$, Nicholas Galitzki ${ }^{4}$, JefFrey Klein ${ }^{4}$, Andrei L. Korotkov ${ }^{9}$, \\ Zhi-Yun Li $^{10}$, Peter G. Martin ${ }^{11}$, Tristan G. Matthews ${ }^{5}$, Lorenzo Moncelsi ${ }^{12}$, Fumitaka Nakamura ${ }^{13}$, \\ Calvin B. Netterfield ${ }^{2,7}$, Giles NovaK ${ }^{5}$, Enzo Pascale ${ }^{3}$, Frédérick Poidevin ${ }^{14,15}$, Fabio P. Santos ${ }^{5}$, Giorgio Savini ${ }^{16}$, \\ Douglas Scott ${ }^{17}$, Jamil A. Shariff ${ }^{2,18}$, Juan Diego Soler ${ }^{19}$, Nicholas E. Thomas ${ }^{20}$, Carole E. Tucker ${ }^{3}$, \\ Gregory S. TUCKer ${ }^{10}$, AND DEREK WARD-ThOMPSON ${ }^{21}$ \\ ${ }^{1}$ Department of Astronomy \& Astrophysics, University of Toronto, 50 St. George Street Toronto, ON M5S 3H4, Canada; ngandil1@jhu.edu \\ ${ }^{2}$ Department of Physics and Astronomy, Johns Hopkins University, 3701 San Martin Drive, Baltimore, Maryland, USA \\ ${ }^{3}$ Cardiff University, School of Physics \& Astronomy, Queens Buildings, The Parade, Cardiff, CF24 3AA, UK \\ ${ }^{4}$ Department of Physics \& Astronomy, University of Pennsylvania, 209 South 33rd Street, Philadelphia, PA, 19104, USA \\ ${ }^{5}$ Center for Interdisciplinary Exploration and Research in Astrophysics (CIERA) and Department of Physics \& Astronomy, Northwestern University, \\ 2145 Sheridan Road, Evanston, IL 60208, USA \\ ${ }^{6}$ Department of Physics, Princeton University, Jadwin Hall, Princeton, NJ 08544, USA \\ ${ }^{7}$ Department of Physics, University of Toronto, 60 St. George Street Toronto, ON M5S 1A7, Canada \\ ${ }^{8}$ Department of Physics and Astrophysics, Nagoya University, Nagoya 464-8602, Japan \\ ${ }^{9}$ Department of Physics, Brown University, 182 Hope Street, Providence, RI, 02912, USA \\ ${ }^{10}$ Department of Astronomy, University of Virginia, 530 McCormick Road, Charlottesville, VA 22904, USA \\ ${ }^{11}$ CITA, University of Toronto, 60 St. George Street, Toronto, ON M5S 3H8, Canada \\ ${ }^{12}$ California Institute of Technology, 1200 E. California Boulevard, Pasadena, CA, 91125, USA \\ ${ }^{13}$ National Astronomical Observatory, Mitaka, Tokyo 181-8588, Japan \\ ${ }^{14}$ Instituto de Astrofsica de Canarias, E-38200 La Laguna, Tenerife, Spain \\ ${ }^{15}$ Universidad de La Laguna, Dept. Astrofísica, E-38206 La Laguna, Tenerife, Spain \\ ${ }^{16}$ Department of Physics \& Astronomy, University College London, Gower Street, London, WC1E 6BT, UK \\ ${ }^{17}$ Department of Physics \& Astronomy, University of British Columbia, 6224 Agricultural Road, Vancouver, BC V6T 1Z1, Canada \\ ${ }_{19}$ Department of Physics, Case Western Reserve University, 2076 Adelbert Road, Cleveland, OH, 44106, USA \\ ${ }^{19}$ Institute d'Astrophysique Spatiale, CNRS (UMR8617) Université Paris-Sud 11, Bâtiment 121, Orsay, France \\ ${ }^{20}$ NASA/Goddard Space Flight Center, Greenbelt, MD 20771, USA \\ ${ }^{21}$ Jeremiah Horrocks Institute, University of Central Lancashire, PR1 2HE, UK \\ Received 2015 December 21; accepted 2016 March 8; published 2016 June 16
}

\begin{abstract}
Polarization maps of the Vela $\mathrm{C}$ molecular cloud were obtained at 250, 350, and $500 \mu \mathrm{m}$ during the 2012 flight of the balloon-borne telescope BLASTPol. These measurements are used in conjunction with $850 \mu \mathrm{m}$ data from Planck to study the submillimeter spectrum of the polarization fraction for this cloud. The spectrum is relatively flat and does not exhibit a pronounced minimum at $\lambda \sim 350 \mu \mathrm{m}$ as suggested by previous measurements of other molecular clouds. The shape of the spectrum does not depend strongly on the radiative environment of the dust, as quantified by the column density or the dust temperature obtained from Herschel data. The polarization ratios observed in Vela $\mathrm{C}$ are consistent with a model of a porous clumpy molecular cloud being uniformly heated by the interstellar radiation field.
\end{abstract}

Key words: dust, extinction - instrumentation: polarimeters - ISM: individual objects (Vela C) - ISM: magnetic fields - submillimeter: ISM - techniques: polarimetric

\section{INTRODUCTION}

The role that magnetic fields play in the process of star formation is not well understood. This question can be addressed by observing the strength and morphology of magnetic fields in the dense molecular clouds where stars form. One important method of observing magnetic fields in star-forming regions is through submillimeter polarimetry. Because dust grains tend to align with their long axes perpendicular to the direction of the local magnetic field, the linearly polarized thermal emission from the dust grains can be used to trace the magnetic field direction in the plane of the sky (see Lazarian 2007 for a review).

The use of polarimetry to probe magnetic fields requires a good understanding of the mechanism by which dust grains align with magnetic fields and how the alignment depends on the local environment. Multiwavelength observations of polarized dust emission can probe the conditions under which dust grains are aligned and test theories of grain alignment mechanisms. The theory of radiative torques (RATs; Dolginov \& Mytrophanov 1976; Lazarian \& Hoang 2007) has become the most favored model for how dust grains align with magnetic fields. This model proposes that an irregularly shaped dust grain with a different cross-section for right- and lefthanded photons will get spun up by unpolarized light in the presence of an anisotropic radiation field, ultimately causing the particle to rotate with its long axis perpendicular to magnetic field lines (see Andersson et al. 2015 for a review).

At visible and near-infrared wavelengths, much has been inferred about the physical properties of dust grains by studying starlight polarization originating from dichroic extinction (Whittet et al. 2001, 2008). In particular, large grains (radii $\geqslant 0.1 \mu \mathrm{m}$ ) are much more efficiently aligned than small grains (Kim \& Martin 1995); amorphous silicate grains are better aligned than carbonaceous grains (including 
polycyclic aromatic hydrocarbons; Smith et al. 2000; Chiar et al. 2006; see Draine 2003). The simplest models for molecular cloud dust (Hildebrand et al. 1999; Bethell et al. 2007) predict submillimeter polarization spectra that are flat to $10 \%-20 \%$. However, the observations to date (Hildebrand et al. 1999; Vaillancourt et al. 2008; Vaillancourt \& Matthews 2012; Zeng et al. 2013) show a polarization fraction with more variation, rising away from a minimum at $350 \mu \mathrm{m}$ (see Figure 4 below).

To explain the rise in the submillimeter spectrum a model in which the colder grains are better aligned than the warmer grains is needed. Bethell et al. (2007) have shown that this can be achieved by applying the RAT model to starless clouds. They model a clumpy molecular cloud structure in which external photons can penetrate deep into the cloud. These photons heat all grains, but the larger grains tend to be cooler, because they are more efficient emitters. At the same time, the mechanism for aligning larger grains is more efficient (Cho \& Lazarian 2005). Therefore, their model predicts that the cooler grains are better aligned and that the polarization spectrum rises with wavelength. However, the predicted submillimeter rise is much shallower than is seen in the published observations. The model also predicts a polarization spectrum rising with wavelength in the far-IR, and so it cannot explain the falling far-IR spectrum that has been observed.

The Balloon-borne Large Aperture Submillimeter Telescope for Polarimetry (BLASTPol) observed the Vela C molecular cloud at 250, 350, and $500 \mu \mathrm{m}$. Multiwavelength observations bracketing the $350 \mu \mathrm{m}$ band provide a new opportunity to study the shape of the polarization spectrum. Vela $\mathrm{C}$ is a relatively nearby molecular cloud, spanning $35 \mathrm{pc}$ at a distance of $d \sim 700 \mathrm{pc}$ (Liseau et al. 1992) and contains $5 \times 10^{4} M_{\odot}$ of dense gas (Yamaguchi et al. 1999). It includes a large number of objects in the early stages of star formation (Netterfield et al. 2009) as well as a bright compact H II region, RCW 36, thus providing an opportunity to study grain alignment in varying radiative conditions. Hill et al. (2011) analyzed Vela C using data from the Herschel HOBYS program, and identified five sub-regions in the cloud, as defined at an $A_{\mathrm{V}}>7$ mag threshold.

This paper presents polarization data in Vela $\mathrm{C}$ from BLASTPol at 250, 350, and $500 \mu \mathrm{m}$, and from Planck at $850 \mu \mathrm{m}$. Section 2 describes the observations of submillimeter polarization in Vela C. Section 3 presents the polarization spectrum of Vela $\mathrm{C}$ and investigates the effect of the radiative environment. Section 4 discusses the implications of this work for theories of dust grain alignment and Section 5 summarizes the results.

\section{OBSERVATIONS AND DATA ANALYSIS}

Polarimetry data at 250, 350, and $500 \mu \mathrm{m}$ were collected during the 2012 flight of BLASTPol (Galitzki et al. 2014; Fissel et al. 2015). BLASTPol was launched on a stratospheric balloon on 2012 December 26 from McMurdo Station, Antarctica and took data for 12.5 days at an altitude of $38.5 \mathrm{~km}$ above sea level. Approximately $43 \mathrm{hr}$ were spent mapping the Vela $\mathrm{C}$ molecular cloud (Figure 1), covering four of the five sub-regions defined by Hill et al. (2011). The data analysis pipeline and instrument characterization are described in Fissel et al. (2015).

The Planck HFI instrument (Planck Collaboration VIII 2016) obtained polarimetry data at $850 \mu \mathrm{m}$ (their $353 \mathrm{GHz}$ band) over

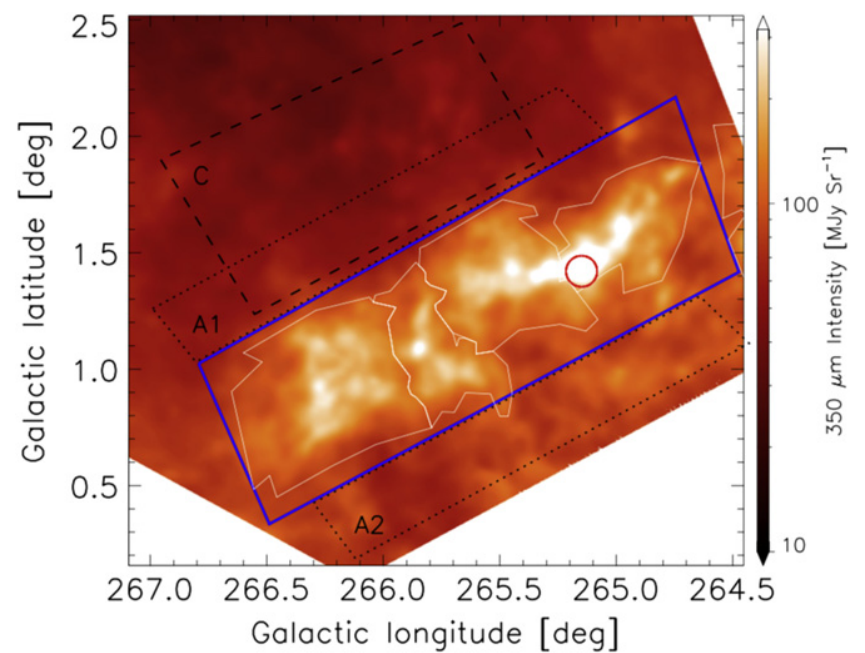

Figure 1. BLASTPol $350 \mu \mathrm{m}$ intensity map of Vela C showing various regions used in the analysis (see the text).

the whole sky (Planck Collaboration Int. XIX 2015). Data from the available map on the Planck Legacy Archive ${ }^{22}$ were regridded to match the BLASTPol maps. To match the resolution of the Planck map, the BLASTPol maps were smoothed to 4 !. 8 . Maps were sampled in pixels of size $2 ! 4$.

To focus the analysis on dust within the Vela $\mathrm{C}$ molecular cloud itself, it is necessary to subtract the emission (in $I, Q$, and $U$ ) from dust along the line of sight and in the extended Vela Molecular Ridge. We adopted two subtraction methods described in Fissel et al. (2015) and their terminology: "conservative" and "aggressive," and a third, "intermediate," for the average of the two. The first uses a single reference region, labelled $\mathrm{C}$ in Figure 1, while the second interpolates a plane between two regions labelled A1 and A2 bracketing the outlined "validity region."

The data were restricted to being inside the Vela $\mathrm{C}$ subregions defined in Hill et al. (2011), because these sightlines are better probes of the polarization structure in the cloud itself. These are shown in white in Figure 1. Data outside these regions are also more sensitive to systematic uncertainties in the method of diffuse emission subtraction. Data from a circular region, shown as a red circle in Figure 1, within $4^{\prime}$ of RCW $36\left(l=265^{\circ} .15, b=1^{\circ} .42\right)$ were also excluded because null tests showed large residuals there.

The polarization fraction, $p$, was calculated at 250, 350, 500, and $850 \mu \mathrm{m}$ from the Stokes parameters $I, Q$, and $U$ as $p=\sqrt{Q^{2}+U^{2}} / I$. Because the polarization amplitude is a positive definite quantity, noise in the $Q$ and $U$ maps will tend to increase the measured polarization. The measured value, $p_{\mathrm{m}}$, was therefore approximately corrected using (Wardle \& Kronberg 1974)

$$
p_{\mathrm{db}}=\sqrt{p_{\mathrm{m}}^{2}-\sigma_{\mathrm{p}}^{2}}
$$

where $p_{\mathrm{db}}$ is the de-biased polarization and $\sigma_{\mathrm{p}}$ is the uncertainty associated with $p_{\mathrm{m}}$. This is a crude approximation, but reasonably accurate for high signal-to-noise ratio measurements (Montier et al. 2015), such as the data analyzed in this work where only $p_{\mathrm{m}} \geqslant 3 \sigma_{\mathrm{p}}$ was used.

\footnotetext{
${ }^{22}$ http://pla.esac.esa.int
} 
The polarization angle, $\psi$, with respect to the $Q, U$ reference frame, was determined as $\psi=\frac{1}{2} \arctan (U, Q)$. The polarization spectrum was studied only where the measurements of $p$ exceeded $3 \sigma_{\mathrm{p}}$ at each of the four wavelengths, and the measured polarization angles at all wavelengths agreed to within $10^{\circ}$. This last cut has been used in previous studies of submillimeter polarization (Vaillancourt \& Matthews 2012) and reduces the probability that data at the different wavelengths are measuring different points along the line of sight.

The number of data points passing the $p \geqslant 3 \sigma_{\mathrm{p}}$ cut depends on the method of diffuse emission subtraction used; however, for all of the subtraction methods none of the remaining data fail the $10^{\circ}$ angle cut. In total the number of data points included in the analysis is 383,405 , and 403 in the case of conservative, aggressive, and intermediate subtraction, respectively, out of a total of 615 measurements lying inside the regions described earlier in this section.

Ratios of $p$ were then calculated relative to $p_{350}$, in order to compare with previous studies, which also normalized their measurements to $350 \mu \mathrm{m}$. Figure 2 shows maps of the polarization ratios $p_{250} / p_{350}, p_{500} / p_{350}$, and $p_{850} / p_{350}$ for the case where the intermediate subtraction method was applied. The $850 \mu \mathrm{m}$ data from Planck have a higher intrinsic noise, resulting in a higher noise level in the $p_{850} / p_{350}$ map.

\section{RESULTS}

\subsection{Median Polarization Ratios}

Figure 3 shows histograms of the three polarization ratios $p_{250} / p_{350}, p_{500} / p_{350}$, and $p_{850} / p_{350}$ for the case of intermediate diffuse subtraction, with the median values indicated. As in previous submillimeter polarization spectrum studies mentioned in Section 1, the distributions of polarization ratios are non-normal, with several outliers. Therefore, the central tendencies of the histograms are better represented by the median values than by the means. The median absolute deviation was used to quantify the scatter in the distribution:

$$
\operatorname{MAD} \equiv \operatorname{median}\left(\left|x-x_{\mathrm{m}}\right|\right),
$$

where $x_{\mathrm{m}}$ is the median value of the measurements $x$. Table 1 lists the medians and MADs for the three polarization ratios using the three diffuse emission subtraction methods. For the intermediate subtraction method, the ratios were $p_{250} /$ $p_{350}=1.02 \pm 0.09, p_{500} / p_{350}=0.93 \pm 0.06$, and $p_{850} /$ $p_{350}=1.07 \pm 0.15$. Although there is a slight minimum at $500 \mu \mathrm{m}$, the median \pm MAD polarization ratios at all of the wavelengths observed are not significantly different from each other, and they are all very close to a flat spectrum, i.e., a ratio of 1.0, as can be visualized in Figure 4 (red triangles). This result does not depend on the method of diffuse emission subtraction.

\subsection{Polarization Ratios from Scatter Plots}

As an alternative, linear fits were performed on three scatter plots of polarization fractions: $250 \mu \mathrm{m}$ versus $350 \mu \mathrm{m}, 500 \mu \mathrm{m}$ versus $350 \mu \mathrm{m}$, and $850 \mu \mathrm{m}$ versus $350 \mu \mathrm{m}$ (Figure 5). For each, the data were fit by minimizing the absolute deviation between the data and a linear model $p_{\lambda}=a_{\lambda}+b_{\lambda} p_{350}$. The slopes, $b_{\lambda}$, of each line constitute a polarization spectrum.
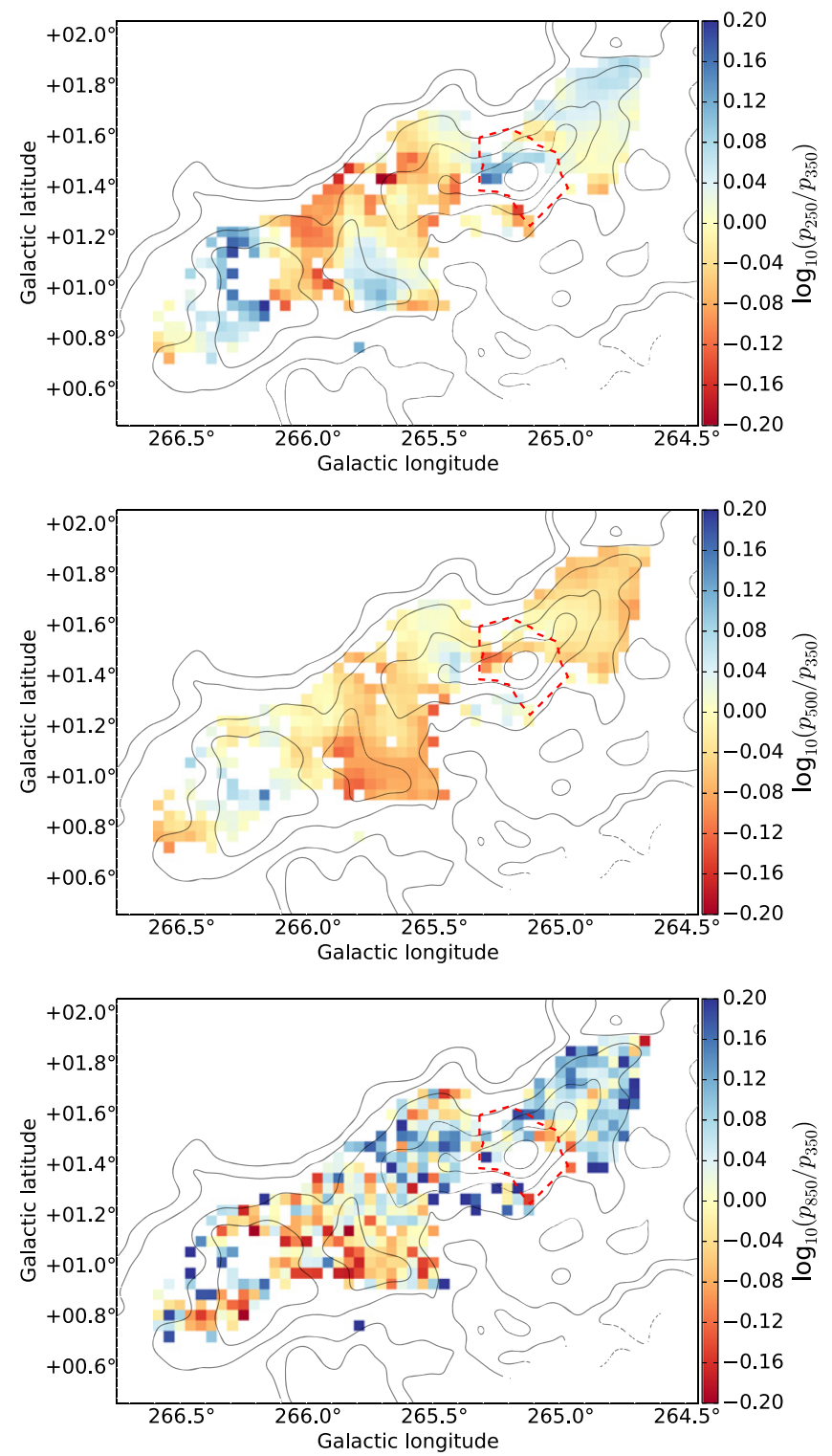

Figure 2. Maps of polarization ratios $p_{250} / p_{350}$ (top), $p_{500} / p_{350}$ (middle) and $p_{850} / p_{350}$ (bottom). The color bar is on a logarithmic scale. Contours show $350 \mu \mathrm{m}$ intensity at levels of $1 \%, 2 \%, 5 \%, 10 \%, 20 \%$, and $50 \%$ of the peak intensity. The red dashed line indicates the region used to isolate the dust being irrradiated by RCW 36 (see Section 3.4).

The least absolute deviation was used rather than a least squares method because it produces a more robust solution, due to its resistance to outliers. The uncertainty on the slopes was calculated using the bootstrapping method with replacement (Press et al. 1992), repeating the fits for each of 10,000 random selections. The standard deviation of the distribution of slopes is used as an estimate of the uncertainty.

Table 2 lists the slopes and their uncertainties for each of the three scatter plots and for each of the three diffuse emission subtraction methods. The spectrum has a minimum at $500 \mu \mathrm{m}$ (see Figure 4, red circles), independent of the method of diffuse emission subtraction. Again, the spectrum is flat to within about $10 \%$.

Comparing the values in Tables 1 and 2 shows that the slopes obtained by fitting to scatter plots are consistent with the medians \pm MADs obtained in the previous section. However, the uncertainties on the slopes in Table 2 are small compared to 


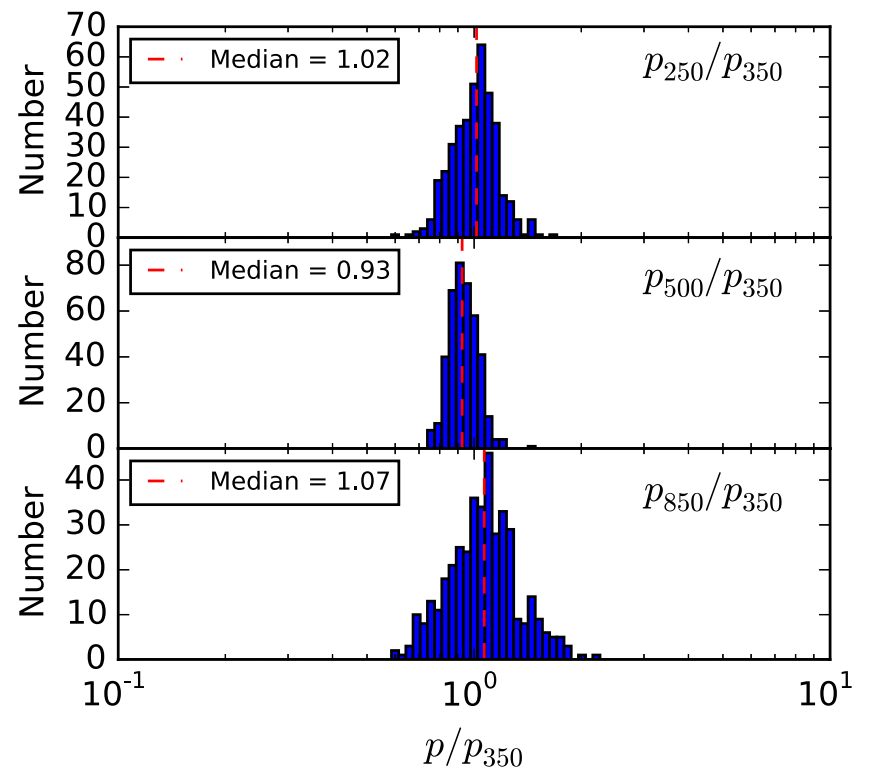

Figure 3. Histograms of the three polarization ratios, using the intermediate diffuse emission subtraction method. Median polarization ratios are indicated by dashed lines.

Table 1

Medians and MADs of Polarization Ratios $\left(p_{\lambda} / p_{350}\right)$

\begin{tabular}{lccc}
\hline \hline $\begin{array}{l}\text { Diffuse Emission } \\
\text { Subtraction Method }\end{array}$ & $250 \mu \mathrm{m}$ & $500 \mu \mathrm{m}$ & $850 \mu \mathrm{m}$ \\
\hline Conservative & $0.97 \pm 0.12$ & $0.93 \pm 0.05$ & $1.04 \pm 0.14$ \\
Aggressive & $1.07 \pm 0.08$ & $0.92 \pm 0.06$ & $1.08 \pm 0.16$ \\
Intermediate & $1.02 \pm 0.09$ & $0.93 \pm 0.06$ & $1.07 \pm 0.15$ \\
\hline
\end{tabular}

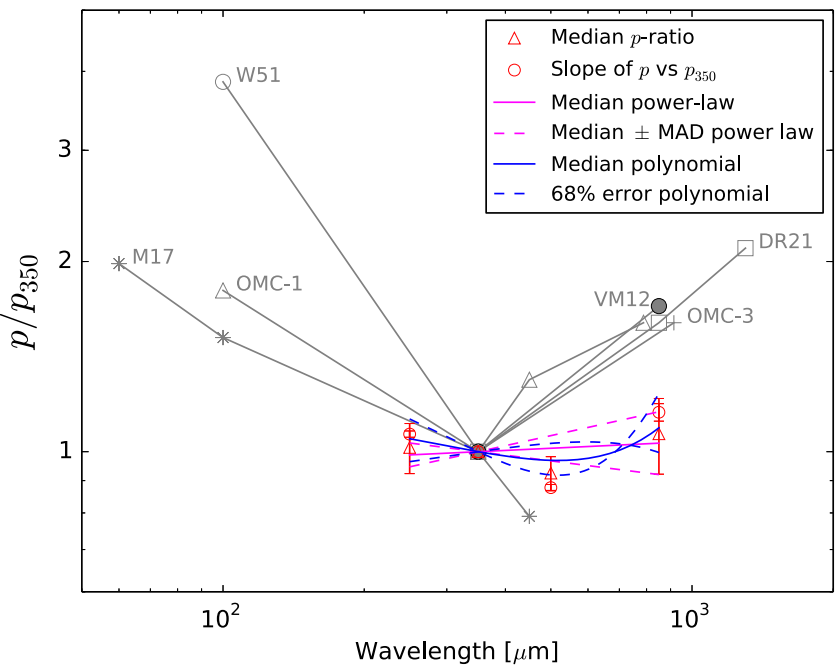

Figure 4. Polarization spectra from previous work (gray), with new Vela $\mathrm{C}$ data added (colors). Points at $850 \mu \mathrm{m}$ separated horizontally for clarity. W51, OMC-1 $p_{100} / p_{350}$, and DR21 $p_{1300} / p_{350}$ from Vaillancourt (2002). All previous measurements of $p_{850} / p_{350}$ from Vaillancourt \& Matthews (2012). The solid circle represents their median ratio for 15 clouds. OMC- $1 p_{450} / p_{350}$ from Vaillancourt et al. (2008). M17 from Zeng et al. (2013). Red triangles are median polarization ratios with MAD error bars. Red circles are best-fit slopes to scatter plots of $p / p_{350}$. Magenta lines are spectra using the power-law fit parameters, and blue lines are spectra using the second-order polynomial fit parameters. Solid lines use the median values of the fit parameters and dashed lines reflect the distribution in the fit parameters (see the text).
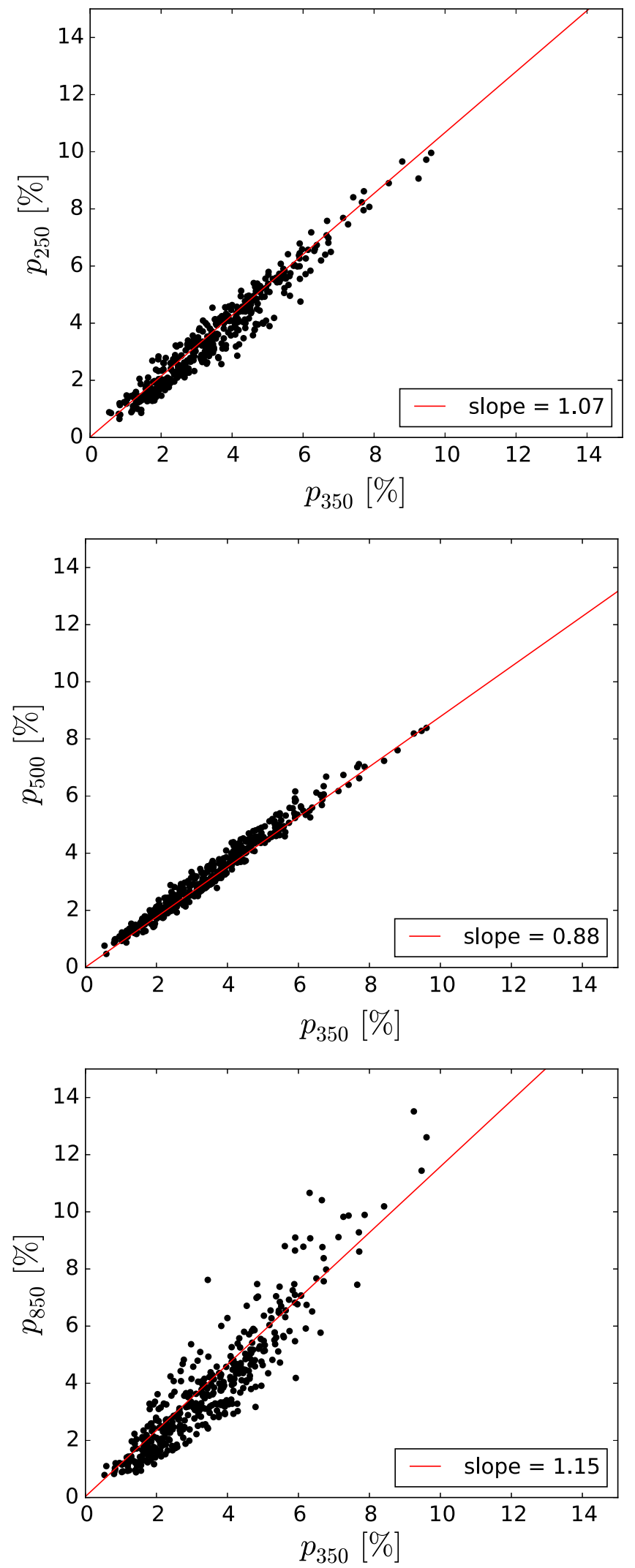

Figure 5. Linear fits to scatter plots of $p_{\lambda}$ vs. $p_{350}$. The red line indicates the best fit to a linear model with the slope indicated.

the values cited in Table 1 for the MADs. These are quite different approaches, here describing the uncertainty in the linear fit to the data, in contrast to the MAD representing the 
Table 2

Slopes of Linear Fits to Scatter Plots of $p_{\lambda}$ vs. $p_{350}$

\begin{tabular}{lccc}
\hline \hline $\begin{array}{l}\text { Diffuse Emission } \\
\text { Subtraction Method }\end{array}$ & $250 \mu \mathrm{m}$ & $500 \mu \mathrm{m}$ & $850 \mu \mathrm{m}$ \\
\hline Conservative & $1.12 \pm 0.01$ & $0.89 \pm 0.01$ & $1.15 \pm 0.04$ \\
Aggressive & $1.04 \pm 0.01$ & $0.87 \pm 0.01$ & $1.19 \pm 0.04$ \\
Intermediate & $1.07 \pm 0.01$ & $0.88 \pm 0.01$ & $1.15 \pm 0.04$ \\
\hline
\end{tabular}

width of the distribution of the ratioed data. It is not surprising that the latter are much larger. In the analogous situation in which the errors were normally distributed, one would calculate the mean and standard deviation about the mean, and then estimate the uncertainty of the mean by dividing the standard deviation by the square root of the number of data.

\subsection{Fits to $p(\lambda)$}

To explore the shape of the spectrum further, fits were performed to $p(\lambda)$ at each individual pixel in the map using the measurements of $p$ at 250,350,500, and $850 \mu \mathrm{m}$. The data were fit to three different functions of $p(\lambda)$; linear

$$
p(\lambda)=a_{l}\left[b_{l}\left(\lambda-\lambda_{0}\right)+1\right]
$$

power law

$$
p(\lambda)=a_{p l}\left(\frac{\lambda}{\lambda_{0}}\right)^{b_{p l}}
$$

and a second-order polynomial

$$
p(\lambda)=a_{2 p}\left[b_{2 p}\left(\lambda-\lambda_{0}\right)^{2}+c_{2 p}\left(\lambda-\lambda_{0}\right)+1\right] .
$$

Here $\lambda_{0}=350 \mu \mathrm{m}$ and in each case $a$ is a normalization constant that is factored out. The linear and power-law fits are different attempts to measure the overall increase or overall decrease of the spectrum in the $250-850 \mu \mathrm{m}$ range. However, in addition, the second-order polynomial fit also allows a spectrum that has a minimum or maximum between 250 and $850 \mu \mathrm{m}$. Although the error bars for $p$ at 250, 350, and $500 \mu \mathrm{m}$ are much smaller than at $850 \mu \mathrm{m}$, the overall uncertainty is dominated by the diffuse emission subtraction method, which affects all four bands. Therefore, each of the bands was given equal weight in the fits to $p(\lambda)$.

Figure 6 shows the results of fitting the power-law and second-order polynomial functions to the data for three example pixels. The linear fit is not shown, because the linear and power-law spectrum look very similar in the $250-850 \mu \mathrm{m}$ range.

The distribution of the fit parameters over all pixels in the Vela $\mathrm{C}$ map was then analyzed. The first three rows of Table 3 list the median values obtained for each of the fit parameters that relate to the spectrum shape $\left(b_{l}, b_{p l}, b_{2 p}\right.$, and $\left.c_{2 p}\right)$, for the three methods of diffuse emission subtraction. Table 3 also contains the median values of $p_{350} / a$, showing how closely the fits match the data at $350 \mu \mathrm{m}$. The typical fractional uncertainty of the measurement of $p_{350}$ is compatible with the MAD. The values of the spectral shape fit parameters are consistent among the three subtraction methods, and their distributions for the case of intermediate subtraction are shown in Figures 7 (powerlaw fit) and 8 (polynomial fit).

The median of the linear fit parameters over the cloud produces a spectrum that gradually rises from $p_{250} / p_{350}=0.99$
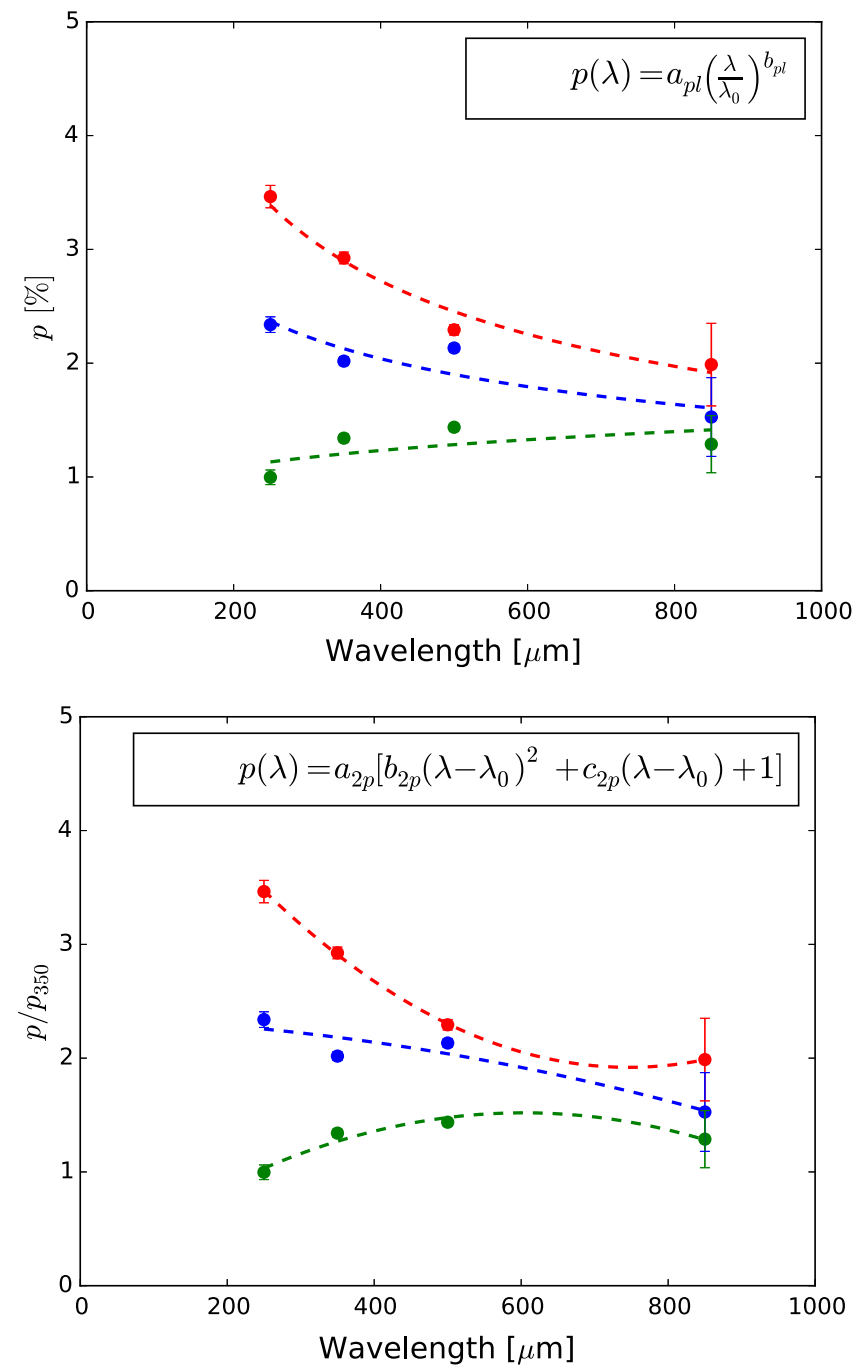

Figure 6. Example fits of $p(\lambda)$ to the data from three pixels at $l=265^{\circ} .79$, $b=1.01$ (red), $l=266^{\circ} .29, b=0.85$ (blue), and $l=266^{\circ} .08, b=0.93$ (green). Top: power-law fit (Equation (4)). Bottom: second-order polynomial fit (Equation (5)).

to $p_{850} / p_{350}=1.03$. The median power-law fit produces a spectrum that is virtually identical to the median linear fit. The second-order polynomial fit was used to probe curvature in the spectrum, looking for a clear minimum or maximum. The median polynomial fit produces a spectrum with a minimum of $p / p_{350} \sim 0.97$ at $\lambda \sim 518 \mu \mathrm{m}$, rising to $p_{250} / p_{350} \sim 1.05$ and $p_{850} / p_{350} \sim 1.10$.

Figure 4 shows polarization spectra over the $250-850 \mu \mathrm{m}$ range plotted using the median $p(\lambda)$ power-law and second-order polynomial fit parameters for the intermediate subtraction method. The dispersion among these fits is illustrated by constructing spectra using the median \pm MAD values of the fit parameters. For the polynomial fit, a scatter plot of $b_{2 p}$ versus $c_{2 p}$ shows that the two terms are highly anti-correlated. A $68 \%$ error ellipse was fit to the distribution; the two endpoints of the major axis of the ellipse were $\left(b_{2 p}, c_{2 p}\right)=\left(-6.1 \times 10^{-7}, 2.9 \times 10^{-4}\right)$ and $\left(b_{2 p}, c_{2 p}\right)=\left(2.9 \times 10^{-6}, 1.0 \times 10^{-3}\right)$. These values were used to construct the dashed blue curves shown in Figure 4. We note that the curves plotted all pass through 1.0 by construction and, from the values of $p_{350} / a$, that the curves typically fit the data to 
Table 3

Median and MADs of $p(\lambda)$ Fit Parameters

\begin{tabular}{|c|c|c|c|c|c|c|c|}
\hline $\begin{array}{l}\text { Diffuse Emission } \\
\text { Subtraction Method }\end{array}$ & $\begin{array}{c}\text { Linear Fit } \\
b_{l}\left(\times 10^{-4}\right)\end{array}$ & $p_{350} / a_{l}$ & $\begin{array}{c}\text { Power-law Fit } \\
\qquad b_{p l}\end{array}$ & $p_{350} / a_{p l}$ & $\begin{array}{l}\text { Polynomial Fit } \\
b_{2 p}\left(\times 10^{-6}\right)\end{array}$ & $c_{2 p}\left(\times 10^{-4}\right)$ & $p_{350} / a_{2 p}$ \\
\hline Conservative & $1.3 \pm 2.7$ & $1.03 \pm 0.06$ & $0.06 \pm 0.13$ & $1.03 \pm 0.06$ & $0.7 \pm 1.4$ & $-1.8 \pm 6.3$ & $1.04 \pm 0.04$ \\
\hline Aggressive & $0.2 \pm 3.1$ & $0.99 \pm 0.03$ & $-0.01 \pm 0.14$ & $0.98 \pm 0.02$ & $1.7 \pm 1.3$ & $-6.6 \pm 5.2$ & $1.01 \pm 0.03$ \\
\hline Intermediate & $0.9 \pm 2.8$ & $1.01 \pm 0.04$ & $0.03 \pm 0.13$ & $1.01 \pm 0.04$ & $1.1 \pm 1.1$ & $-3.7 \pm 5.0$ & $1.03 \pm 0.03$ \\
\hline Intermediate $\left(p / p_{350}\right)$ & $-2.9 \pm 3.5$ & $\ldots$ & $-0.21 \pm 0.13$ & $\ldots$ & $2.3 \pm 0.6$ & $-8.5 \pm 2.5$ & $\ldots$ \\
\hline
\end{tabular}

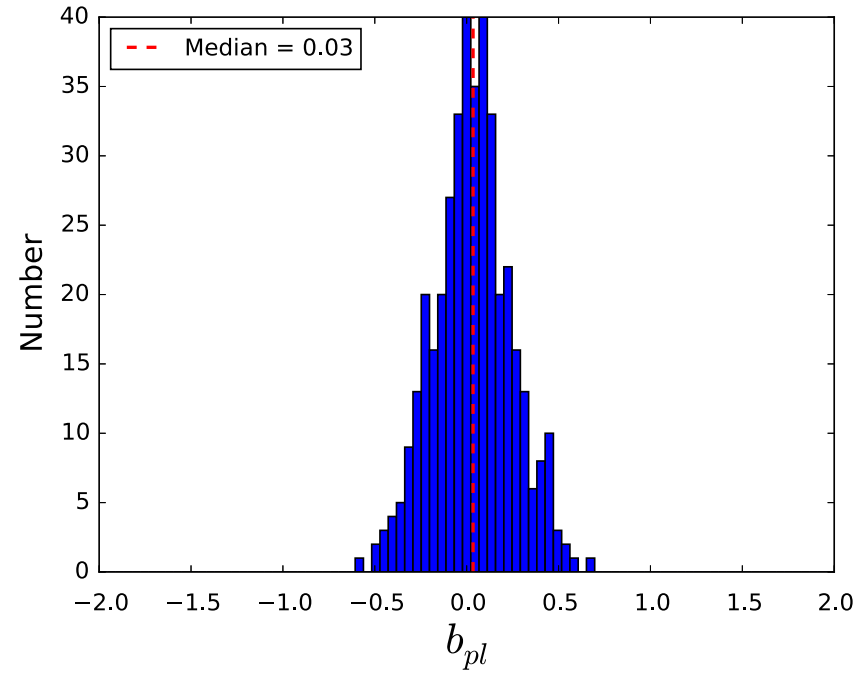

Figure 7. Distribution of power-law exponent $b_{p l}$ from Equation (4).

within $4 \%$ at $350 \mu \mathrm{m}$, which is about the size of the symbols plotted there.

An alternative method is to fit to the slopes $p / p_{350}$ from Table 2, using their associated uncertainties as weights in the fit. Equations (3)-(5) were modified to the normalized form with no " $a$ " fitting parameter and there was no $350 \mu \mathrm{m}$ data point. The remaining fit parameters obtained using this method are listed at the bottom of Table 3. The first two types of fits to $p$ have negative slopes reflecting the down-weighting of the $850 \mu \mathrm{m}$ data. However, the polynomial fit is not greatly changed, with a minimum of $p / p_{350} \sim 0.92$ at $\lambda \sim 535 \mu \mathrm{m}$, rising to $p_{250} / p_{350} \sim 1.10$ and $p_{850} / p_{350} \sim 1.15$.

\subsection{Effect of Environment}

The environment in the Vela $\mathrm{C}$ molecular cloud can be represented quantitatively by column density of hydrogen nuclei, $N\left[\mathrm{~cm}^{-2}\right]$ and dust temperature, $T[\mathrm{~K}]$. These quantities were derived from modified blackbody SED fits to Herschel data at $160,250,350$, and $500 \mu \mathrm{m}$, assuming a dust spectral index $\beta=2$. The methodology is described in detail in Fissel et al. (2015). For this analysis, the Herschel maps were smoothed to $5^{\prime}$ before the SED fitting.

To investigate whether the shape of the polarization spectrum depends on environment, the $p(\lambda)$ fit parameters obtained in Section 3.3 were plotted versus $N$ and $T$. The results are shown in Figure 9 for the power-law and polynomial fit parameters. The data were binned in $N$ and $T$, and the mean value of each parameter plotted for each bin. No trend is seen with $N$, i.e., for all of the $N$ bins, the average value stays within the median \pm MAD listed in Table 3 . No trend is seen over most of the $T$ bins, but for the sparse data at high $T$ the
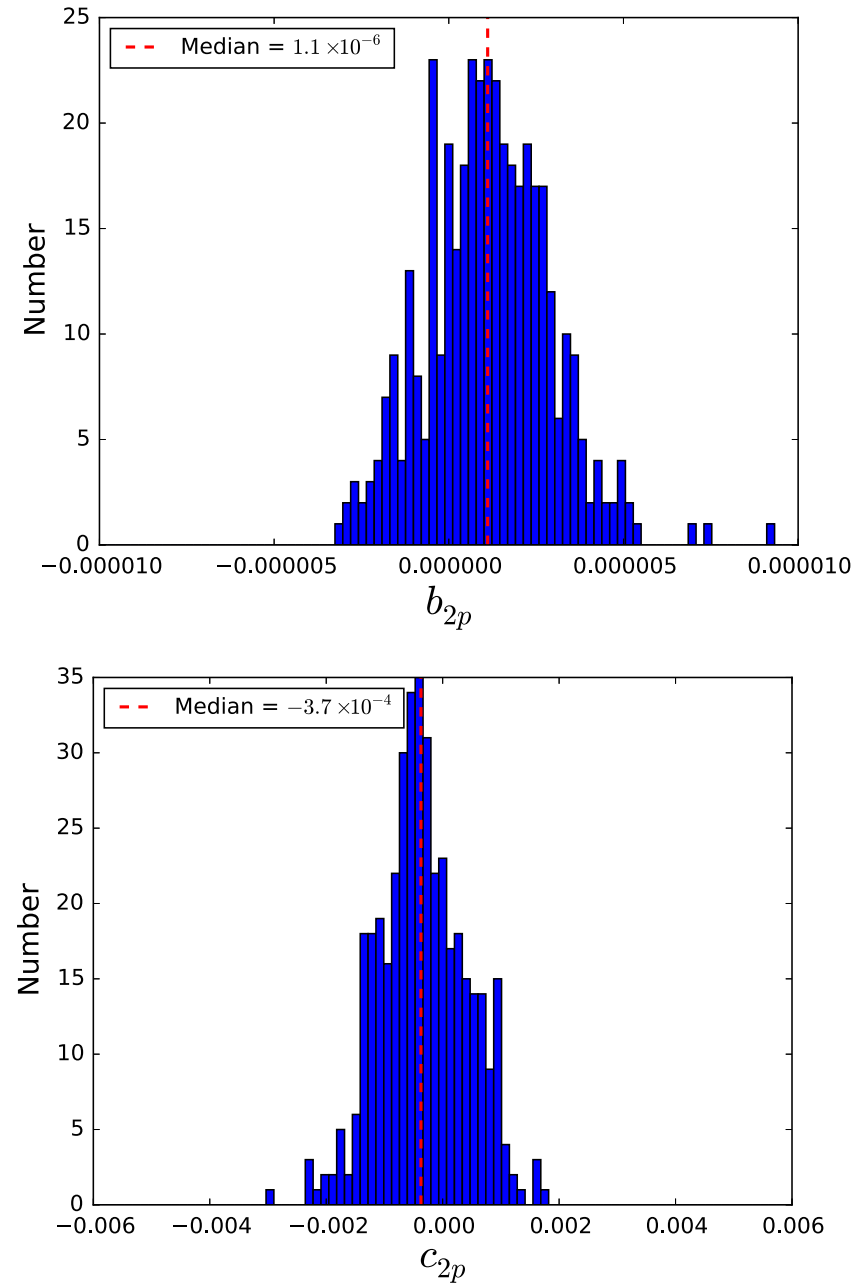

Figure 8. Distributions of polynomial fit parameters $b_{2 p}$ (top) and $c_{2 p}$ (bottom) from Equation (5).

polynomial fit gives a slightly higher second-order coefficient, with a correspondingly lower value of the anti-correlated firstorder coefficient.

The high $T$ data come from the region of the map around the compact $\mathrm{H}$ II region $\mathrm{RCW} 36$, and so the effect of environment was also examined by separating the cloud into two regions. In one region, the dust is heated by RCW 36, and in the other it is heated by the interstellar radiation field (ISRF). Fissel et al. (2015) describe how the separation of the two populations was done, based on the observation of a clear trend of decreasing $T$ with increasing $N$ for dust heated by the ISRF. Data that do not lie along this trend line are from sightlines heated by RCW 36. The closed curve that separates the two regions is shown in Figure 2. The numbers of data points were 18 (RCW 36) and 


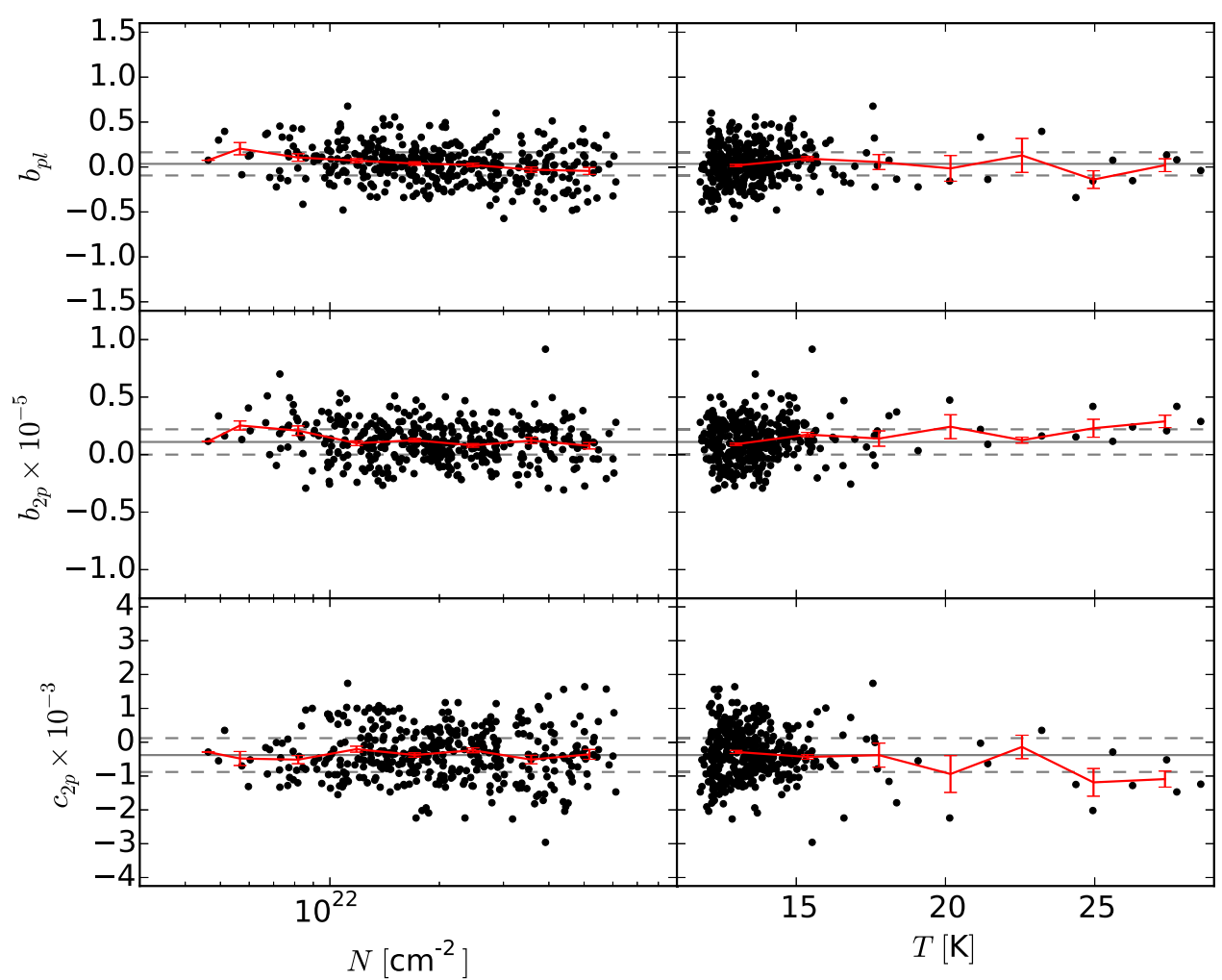

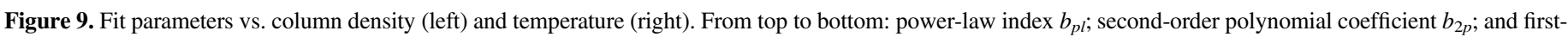

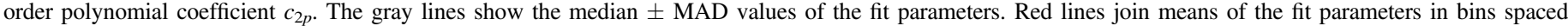
logarithmically in $N$ and linearly in $T$. Error bars indicate the standard deviation of each bin.

385 (ISRF). The same methods were then applied to each of the two regions; the results are listed in Table 4.

The results for the ISRF-heated sightlines are virtually identical to those for the Vela $\mathrm{C}$ cloud as a whole. The results for the two groups are also consistent with each other within their uncertainties. Figure 10 shows the spectrum for the RCW 36-heated sightlines.

\section{DISCUSSION}

Figure 4 summarized the polarization spectrum measured for Vela C, using all of the methods described in the previous sections. Also shown for comparison are the polarization spectra of other molecular clouds reported in previous work (see the caption). In combination, the measurements of the polarization spectra in other clouds are suggestive of a minimum at $\lambda \sim 350 \mu \mathrm{m}$. However, the minimum is actually seen directly in only one individual source, OMC-1. The data for M17 are monotonic through $350 \mu \mathrm{m}$ and all other sources lack sufficient wavelength coverage to show this $\mathrm{V}$ shape, or not, having polarization spectra that rise away from $350 \mu \mathrm{m}$ on either the longward or shortward side, but not both. In contrast, the BLASTPol data in Vela $\mathrm{C}$ are consistent with a flat spectrum (i.e., $p / p_{350} \sim 1.0$ ) in the $250-850 \mu \mathrm{m}$ range, regardless of the method of measuring the polarization spectrum.

Shortward of $350 \mu \mathrm{m}$, the Vela $\mathrm{C}$ data cannot be directly compared to other clouds, because no previous measurements were made at $250 \mu \mathrm{m}$. Measurements at 60 and $100 \mu \mathrm{m}$ generally indicate a steep decrease in the polarization spectrum from 60 to $350 \mu \mathrm{m}$, but the $250 \mu \mathrm{m}$ measurement in Vela $\mathrm{C}$ does not follow this trend.
Table 4

Results for Sightlines Heated by RCW 36 vs. Sightlines Heated by the ISRF

\begin{tabular}{lcc}
\hline \hline Measurement Quantity & RCW 36 & ISRF \\
\hline Median $p_{250} / p_{350}$ & $1.17 \pm 0.11$ & $1.01 \pm 0.09$ \\
Median $p_{500} / p_{350}$ & $0.91 \pm 0.05$ & $0.93 \pm 0.06$ \\
Median $p_{850} / p_{350}$ & $1.10 \pm 0.16$ & $1.06 \pm 0.15$ \\
Slope of $p_{250}$ vs $p_{350}$ & $0.99 \pm 0.21$ & $1.07 \pm 0.01$ \\
Slope of $p_{500}$ vs $p_{350}$ & $1.03 \pm 0.05$ & $0.87 \pm 0.01$ \\
Slope of $p_{850}$ vs $p_{350}$ & $1.24 \pm 0.20$ & $1.15 \pm 0.04$ \\
$p(\lambda)$ linear slope $b_{l}\left(\times 10^{-4}\right)$ & $-1.0 \pm 2.9$ & $1.0 \pm 2.8$ \\
$p(\lambda)$ power-law exponent $b_{p l}$ & $-0.09 \pm 0.12$ & $0.037 \pm 0.13$ \\
$p(\lambda)$ polynomial fit $b_{2 p}\left(\times 10^{-6}\right)$ & $2.3 \pm 1.3$ & $1.1 \pm 1.1$ \\
$p(\lambda)$ polynomial fit $c_{2 p}\left(\times 10^{-4}\right)$ & $-12 \pm 6.7$ & $-3.6 \pm 4.9$ \\
\hline
\end{tabular}

There are previous measurements of the spectrum at $850 \mu \mathrm{m}$ by Vaillancourt \& Matthews (2012) that can be compared with the Vela $\mathrm{C}$ measurement. There, the spectrum in other molecular clouds is again steeper than in Vela $\mathrm{C}$, rising to a ratio of $p_{850} / p_{350}=1.6-1.7$, compared to about 1.1 in Vela $\mathrm{C}$.

The putative V-shaped far-IR decrease and submillimeter rise seen by other experiments have yet to be connected to a theoretical dust model. Hildebrand et al. (1999) and Vaillancourt et al. (2008) argue that the observed behavior is not consistent with a simple isothermal dust model, but requires multiple grain populations, where each population's polarization efficiency is correlated with either the dust temperature or spectral index.

The Draine \& Fraisse (2009) models produce polarization spectra that rise from $p_{250} / p_{350} \sim 0.9$ to $p_{850} / p_{350} \sim 1.0-1.3$, depending on the composition, shapes, and alignment of the 


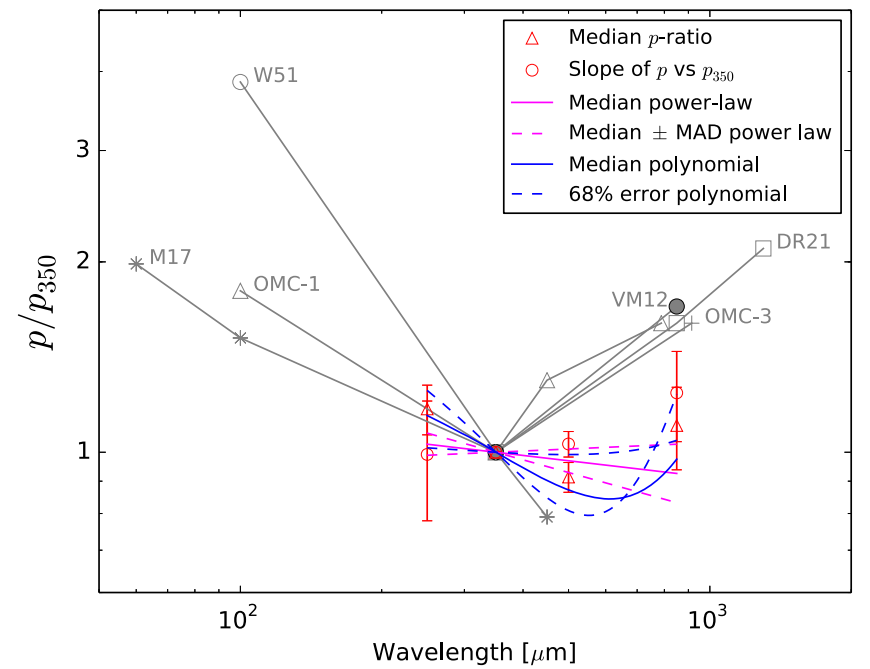

Figure 10. Polarization spectra for RCW 36-heated sightlines in Vela C. See caption to Figure 4 for an explanation of the symbols used.

grain mixture. However, their models apply to the diffuse interstellar medium $\left(A_{\mathrm{V}}<4\right.$ mag), not to dense molecular clouds such as Vela C. The Bethell et al. (2007) molecular cloud model is more relevant to this study and also predicts a spectrum that gradually rises from $p_{250} / p_{350} \sim 0.9$ to $p_{850} / p_{350}$ $\sim 1.1$.

A comparison of the Vela $\mathrm{C}$ data with the spectrum predicted by Bethell et al. (2007) over the 350-850 $\mu \mathrm{m}$ range is shown in Figure 11. The Vela $\mathrm{C}$ data are shown as the average degree of polarization at each wavelength (total polarized intensity, $P$, divided by total intensity, I), normalized to $350 \mu \mathrm{m}$. The data were restricted to the region heated by the ISRF. Unlike the model, these Vela $\mathrm{C}$ data (as in Figure 10) show a slight minimum at $500 \mu \mathrm{m}$, rather than a rise from 250 to $850 \mu \mathrm{m}$. However, the data resemble the model more closely than the previous observations of other molecular clouds. While the model spectrum is fairly flat longward of $\lambda \sim 300 \mu \mathrm{m}$, it falls precipitously at wavelengths shorter than what BLASTPol measured. Future measurements by experiments like HAWC+ (Dowell et al. 2013), a polarimeter for SOFIA operating at $50-220 \mu \mathrm{m}$, would help to constrain the far-IR part of the polarization spectrum.

While Bethell et al. (2007) work under the assumption of starless clouds, in real molecular clouds there exist embedded stars that provide an additional source of photons. The part of the spectrum that increases toward the far-IR could be due to embedded sources both heating the dust grains and leading to a higher alignment efficiency through RATs (Vaillancourt \& Matthews 2012; Zeng et al. 2013). The absence of a spectrum that increases below $350 \mu \mathrm{m}$ in Vela $\mathrm{C}$ might be due to the early evolutionary state of the cloud. However, future work at high resolution could look for the effect of embedded sources in Vela $\mathrm{C}$ by measuring the polarization spectrum toward sightlines of known protostars.

A major difference underlying the data compared is that the Vela $\mathrm{C}$ data were obtained from balloon-borne and space-based observatories while the results from previous works (shown in Figures 4, 10, and 11) use a combination of data from airborne (12.5 km altitude) and ground-based observatories (longward of $100 \mu \mathrm{m}$, the data are all from the ground). Because they are observing through the Earth's atmosphere, these experiments

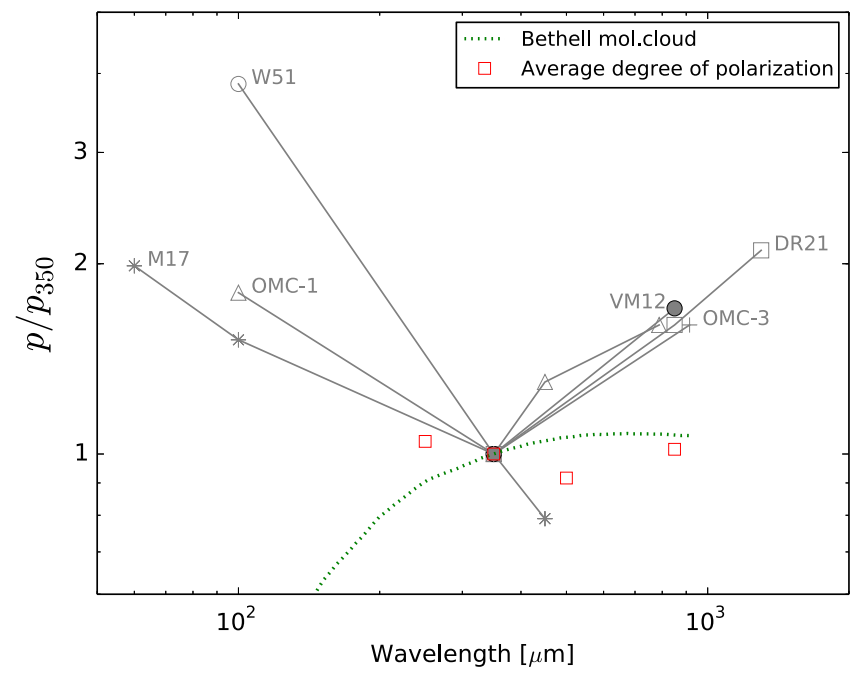

Figure 11. Comparison with the predicted polarization spectrum from Bethell et al. (2007), represented by the green dashed line. Red squares represent the total polarized fraction of the Vela C data, normalized to $350 \mu \mathrm{m}$.

are flux limited to very bright dense parts of molecular clouds. In contrast, the Vela $\mathrm{C}$ map probes a wider area of colder $(11-15 \mathrm{~K})$ dust in relatively quiescent regions. One way of quantifying the difference between the environment of the dust in Vela $\mathrm{C}$ and that in the clouds observed from the ground is by using the $850 \mu \mathrm{m}$ intensity. For the data used by Vaillancourt \& Matthews (2012) to study 17 molecular clouds, the median intensity at $850 \mu \mathrm{m}$ is $637 \mathrm{MJy} \mathrm{sr}^{-1}$, with an interquartile range of 300-1327 $\mathrm{MJy} \mathrm{sr}^{-1}$. In the Vela $\mathrm{C}$ data used here, the median intensity at $850 \mu \mathrm{m}$ is $9.1 \mathrm{MJy} \mathrm{sr}^{-1}$, with an interquartile range of $6.5-14.1 \mathrm{MJy} \mathrm{sr}^{-1}$.

The part of Vela $\mathrm{C}$ being radiated by RCW 36 is the most comparable to the bright regions of clouds observed by other experiments. However, in RCW, 36-heated sightlines alone, the median $850 \mu \mathrm{m}$ intensity is still only $20.2 \mathrm{MJy} \mathrm{sr}^{-1}$, with an interquartile range of 12.4-25.4 $\mathrm{MJy} \mathrm{sr}^{-1}$. When the dust being irradiated by RCW 36 is analyzed separately, the various methods of measuring the shape of the polarization spectrum still give results that are consistent with a flat spectrum (Figure 10), in contrast to the V-shape. It is worth noting that the region closest to RCW 36 was excluded from analysis (see Section 2), and the shape of the spectrum might be changed by adding those data points if such data were available.

Although the radiative environment of the dust in Vela $\mathrm{C}$ was quantified by $N$ and $T$, it is possible that more complex metrics are needed. For example, the RAT mechanism predicts that grain alignment is highly dependent on the anisotropy of the radiation field. Indeed, Andersson \& Potter (2010) and Vaillancourt \& Andersson (2015) find a dependence of the polarization fraction on the relative angle between the radiation field anisotropy and the magnetic field direction. One might carry out such a test in Vela C using the peak in the RCW 36 intensity to define a relative angle of radiation anisotropy, but this has not been investigated given the low spatial resolution of the data.

\section{SUMMARY}

A total of 403 measurements were made by BLASTPol in the Vela C molecular cloud, at 250, 350, and $500 \mu \mathrm{m}$. These were analyzed with Planck measurements at $850 \mu \mathrm{m}$. The data 
were used to measure the polarization spectrum using several methods, including the median polarization ratios, the slopes of $p$ versus $p_{350}$ scatter plots, and fits to functions $p(\lambda)$. All methods indicate that the spectrum is quite flat, especially compared to the $\mathrm{V}$-shaped spectrum suggested by previous observations in other molecular clouds. The polarization fraction remains relatively constant from 250 to $850 \mu \mathrm{m}$ and does not depend significantly on the environment of the cloud, as quantified by $N$ or $T$ or by the source of irradiation (RCW 36 or ISRF). From 250 to $850 \mu \mathrm{m}$ the spectrum in Vela $\mathrm{C}$ appears consistent with the predicted spectrum from Bethell et al. (2007) for a starless molecular cloud; measurements at shorter wavelengths would provide further constraints.

The BLASTPol collaboration acknowledges support from NASA (through grant numbers NAG5-12785, NAG5-13301, NNGO-6GI11G, NNX0-9AB98G, and the Illinois Space Grant Consortium), the Canadian Space Agency, the Leverhulme Trust through the Research Project Grant F/00 407/BN, Canada's Natural Sciences and Engineering Research Council, the Canada Foundation for Innovation, the Ontario Innovation Trust, and the US National Science Foundation Office of Polar Programs. Based on observations obtained with Planck (http:/ / www.esa.int/Planck), an ESA science mission with instruments and contributions directly funded by ESA Member States, NASA, and Canada. C.B.N. also acknowledges support from the Canadian Institute for Advanced Research. F.P.S. is supported by the CAPES grant 2397/13-7. F.P. thanks the Spanish Ministry of Economy and Competitiveness (MINECO) under the Consolider-Ingenio project CSD2010-00064. P.A. is supported through Reach for the Stars, a GK-12 program supported by the National Science Foundation under grant DGE-0948017. Finally, we thank the Columbia Scientific Balloon Facility staff for their outstanding work.

\section{REFERENCES}

Andersson, B.-G., Lazarian, A., \& Vaillancourt, J. E. 2015, ARA\&A, 53, 501 Andersson, B.-G., \& Potter, S. B. 2010, ApJ, 720, 1045

Bethell, T. J., Chepurnov, A., Lazarian, A., \& Kim, J. 2007, ApJ, 663, 1055 Chiar, J. E., Adamson, A. J., Whittet, D. C. B., et al. 2006, ApJ, 651, 268

Cho, J., \& Lazarian, A. 2005, ApJ, 631, 361

Dolginov, A. Z., \& Mytrophanov, I. G. 1976, Ap\&SS, 43, 257

Dowell, C. D., Staguhn, J., Harper, D. A., et al. 2013, BAAS, 221, 345.14

Draine, B. T. 2003, ARA\&A, 41, 241

Draine, B. T., \& Fraisse, A. A. 2009, ApJ, 696, 1

Fissel, L. M., Ade, P. A. R., Angilè, F. E., et al. 2015, ApJ, submitted (arXiv:1509.05298)

Galitzki, N., Ade, P. A. R., Angilè, F. E., et al. 2014, Proc. SPIE, 9145, 91450R

Hildebrand, R. H., Dotson, J. L., Dowell, C. D., Schleuning, D. A., \& Vaillancourt, J. E. 1999, ApJ, 516, 834

Hill, T., Motte, F., Didelon, P., et al. 2011, A\&A, 533, A94

Kim, S.-H., \& Martin, P. G. 1995, ApJ, 444, 293

Lazarian, A. 2007, JQSRT, 106, 225

Lazarian, A., \& Hoang, T. 2007, MNRAS, 378, 910

Liseau, R., Lorenzetti, D., Nisini, B., Spinoglio, L., \& Moneti, A. 1992, A\&A, 265,577

Montier, L., Plaszczynski, S., Levrier, F., et al. 2015, A\&A, 574, A135

Netterfield, C. B., Ade, P. A. R., Bock, J. J., et al. 2009, ApJ, 707, 1824

Planck Collaboration VIII 2016, A\&A, in press (arXiv:1502.01587v2)

Planck Collaboration Int. XIX 2015, A\&A, 576, A104

Press, W. H., Teukolsky, S. A., Vetterling, W. T., \& Flannery, B. P. 1992, Numerical Recipes in C: The Art of Scientific Computing (2nd ed.; Cambridge: Cambridge Univ. Press)

Smith, C. H., Wright, C. M., Aitken, D. K., Roche, P. F., \& Hough, J. H. 2000, MNRAS, 312, 327

Vaillancourt, J. E. 2002, ApJS, 142, 53

Vaillancourt, J. E., \& Andersson, B.-G. 2015, ApJL, 812, L7

Vaillancourt, J. E., Dowell, C. D., Hildebrand, R. H., et al. 2008, ApJL, 679, L25

Vaillancourt, J. E., \& Matthews, B. C. 2012, ApJS, 201, 13

Wardle, J. F. C., \& Kronberg, P. P. 1974, ApJ, 194, 249

Whittet, D. C. B., Gerakines, P. A., Hough, J. H., \& Shenoy, S. S. 2001, ApJ, 547,872

Whittet, D. C. B., Hough, J. H., Lazarian, A., \& Hoang, T. 2008, ApJ, 674, 304

Yamaguchi, N., Mizuno, N., Saito, H., et al. 1999, PASJ, 51, 775

Zeng, L., Bennett, C. L., Chapman, N. L., et al. 2013, ApJ, 773, 29 